\title{
Globaler Wandel - Verlust an Ressourcen?
}

\author{
Workshop-Tagung Geoökologie, 19.-21. November 2010 in Freiberg
}

\author{
Viridiana Alcántara Cervantes · Andreas Schellenberger · Robert Sieland · Gerald Schmidt
}

Eingegangen: 27. August 2010/Akzeptiert: 31. August 2010/Online veröffentlicht: 25. September 2010

(C) Springer-Verlag 2010

\section{Abendvortrag am Freitag, dem 19. November 2010}

Um 19:00 Uhr wird Dr. Harry Lehmann, Leiter des Fachbereichs I „Umweltplanung und Nachhaltigkeit“ des Umweltbundesamtes in Dessau, die Jahresveranstaltung des VGöD eröffnen. Sein Vortrag wird dem Leitthema der WorkshopTagung gewidmet sein. Die interessierte Öffentlichkeit ist herzlich eingeladen, den Abendvortrag zu besuchen.

Der restliche Abend steht zur freien Verfügung. Erfahrungsgemäß finden sich kurz nach der letzten Frage an den Referenten die ersten Gruppen in den benachbarten Gaststätten ein - sei es, um das Gehörte zu diskutieren, oder auch nur, um ein Wiedersehen zu feiern.

\section{Ein Appetizer, dann die Workshops am Samstag, dem 20. November 2010}

Am eigentlichen Workshoptag wird Dr. Klaus Töpfer - den älteren Semestern als früherer Bundesminister für Umwelt, Naturschutz und Reaktorsicherheit bekannt - dem Audito-

\section{V.A. Cervantes}

KIT, Karlsruhe, 76133 Karlsruhe, Deutschland

E-Mail: viridiana.alcantara@geooekologie.de

\section{A. Schellenberger}

Bundesamt für Umwelt, 3003 Bern, Schweiz

E-Mail: andreas.schellenberger@geooekologie.de

R. Sieland

TU Bergakademie Freiberg, 09596 Freiberg, Deutschland

E-Mail: robert.sieland@geo.tu-freiberg.de

G. Schmidt $(\bowtie)$

Karl-Winnacker-Institut, DECHEMA e. V.,

60486 Frankfurt am Main, Deutschland

E-Mail: gerald.schmidt@geooekologie.de rium rasch jegliche Morgenmüdigkeit austreiben. Gestützt auf sein langjähriges Engagement für eine nachhaltige Entwicklung und eine in vielen Reden erprobte Rhetorik dürfte er höchst spannend und unterhaltsam in und durch den Themenkomplex „Globaler Wandel“ führen.

Im Anschluss an das Einstiegsreferat geben die Workshopleiter (s.u.) jeweils einen Impulsvortrag zu den drei Schwerpunktbereichen Boden, Wasser und Biodiversität. Sie werden um einen gelungenen Spannungsbogen bemüht sein, denn jeder Tagungsteilnehmer/jede Tagungsteilnehmerin kann sich nach diesem kondensierten Einstieg in das spezifische Fachgebiet für einen der parallel stattfindenden Workshops entscheiden.

Der Workshop Boden wird von Prof. Dr. Reinhard Hüttl (GeoForschungsZentrum Potsdam, Uni Cottbus) und Dipl.Geoökol. Constance Bornkampf (Doktorandin am Institut für Bohrtechnik und Fluidbergbau an der TU Bergakademie Freiberg) geleitet.

Prof. Dr. Tillmann Buttschardt (Institut für Landschaftsökologie, Uni Münster) und Dr. Sonja Knapp (Helmholtz Zentrum für Umweltforschung, Halle, Department Biozönoseforschung) übernehmen den Workshop für Biodiversität.

Prof. Dr. Erwin Zehe (Institut für Wasser und Umwelt, Uni München) und Dipl.-Geoökol. Daniel Klein (Wiss. Mitarbeiter am Institut für Siedlungswasserwirtschaft der TU Braunschweig) gestalten den Workshop Wasser.

In den Workshops sollen (themenspezifisch) verschiedene Aspekte des globalen Wandels erörtert und die vielfältigen Verknüpfungen zwischen verschiedenen Umweltfaktoren inklusive des anthropogenen Einflusses zur Sprache gebracht werden. Die Leiter ermuntern die Teilnehmer ausdrücklich, eigene Ideen einzubringen und mit den anwesenden Fachleuten die bekannten - und ebenso die vielleicht noch nicht so klar absehbaren - Probleme im Mensch-Umwelt-System zu analysieren. 
Abb. 1 Bergwerk, „Reiche Zeche" in Freiberg, Exkursion 1. Foto: Dr. Jürgen Weyer (Institut für Bergbau und Spezialtiefbau, TU Freiberg)

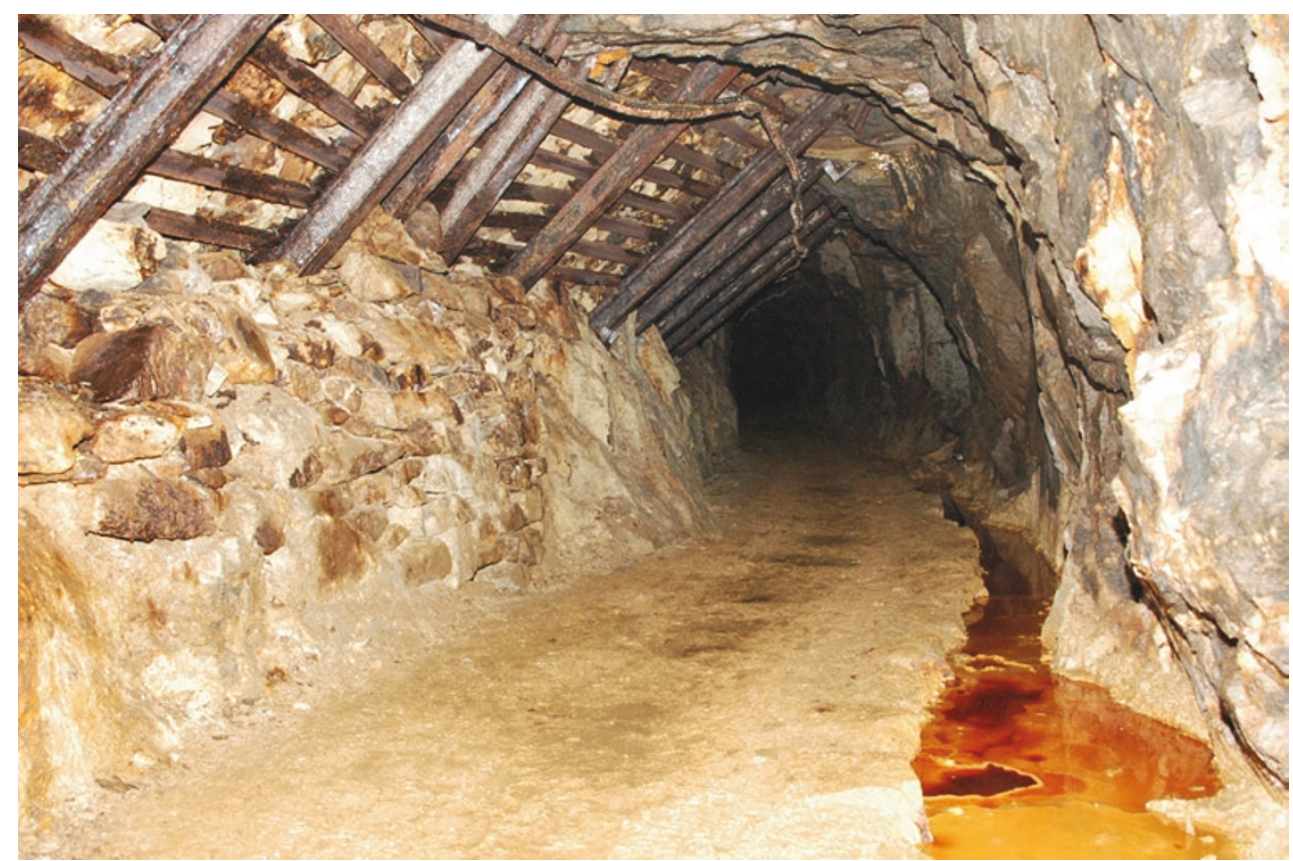

Nach den Workshops treffen sich alle Teilnehmer im Plenum wieder und diskutieren gemeinsam die erzielten Ergebnisse. Die Kerninhalte werden im Schwerpunktthema im Forum der Geoökologie Ausgabe 1/2011 aufgegriffen.

\section{Jahreshauptversammlung des VGöD}

Um 17.15 Uhr findet die Jahreshauptversammlung des VGöD statt. Dieses Jahr stehen wieder Wahlen im Vorstand an, und einige Posten sind neu zu vergeben.

\section{Geselliger Abend}

Am Samstagabend erwartet uns im Freiberger Brauhof ein wohl verdientes bzw. wohlverdientes Buffet - je nachdem, ob der Tag ebenso produktiv wie lang war. Wie auch immer, an Vegetarier ist gedacht, und die Organisatoren haben versichert, auch für musikalische Unterhaltung zu sorgen - die besten Voraussetzungen also für eine schwungvolle Vernetzung von Göks, Geckos ... und was dann sonst noch herumspringt!

\section{Exkursionen am Sonntag, dem 21. November 2010}

Bevor es zurück nach Hause geht, werden den Tagungsteilnehmern drei spannende halbtägige Exkursionen zur
Auswahl angeboten. Eine gute Gelegenheit, Freiberg, seine Umgebung und seine Vergangenheit näher kennenzulernen:

- der Schacht „Reiche Zeche“, der bei einer Erlebnisführung einen Einblick in die Geschichte des Bergbaus in Freiberg bietet (Abb. 1),

- die Forschungsstation Oberbärenburg, abgelegen und doch nah genug, dass Luftschadstoffe aus den Industrieund Ballungsräumen Sachsens gemessen werden können und

- die Ausstellung Terra Mineralia im Schloss Freudenstein, eine der bedeutendsten und wertvollsten Mineralsammlungen der Welt, samt Stadtführung.

\section{Anmeldung}

Alle Details zum Programm und zur Anmeldung sind auf der Homepage des VGöD (www.geooekologie.de) zu finden. Der Verband und Prof. Dr. Jörg Matschullat, Wolfram Canzler und Robert Sieland von der TU Bergakademie Freiberg laden alle Interessenten herzlich zur Jahrestagung ein und freuen sich auf Ihre Anwesenheit!

Ihr Kontakt zum VGöD: Verband für Geoökologie in Deutschland e.V., Hauptgeschäftsstelle, Bianca Schiffner, Alexanderstr. 9, 95444 Bayreuth; Tel.: +49-921-72159215; Fax: +49-921-851497, E-Mail: vgoed@geooekologie.de, www.geooekologie.de 